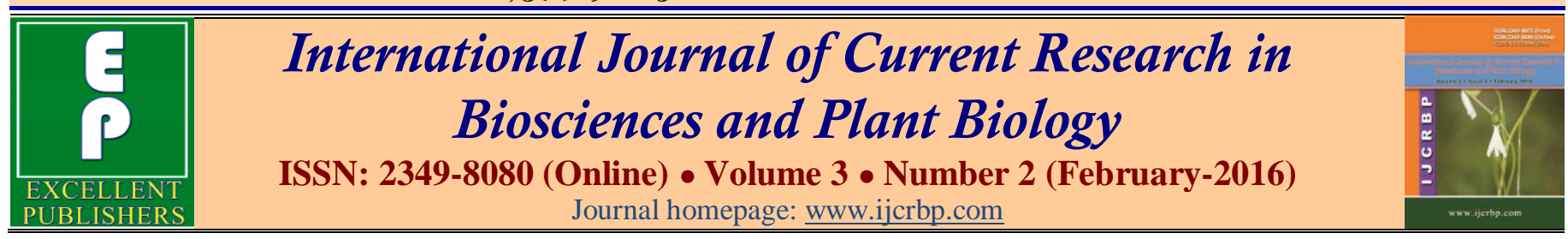

\title{
Floral Diversity of Vaigai River in Thiruppuvanam Region of Sivagangai District, Tamil Nadu, Southern India
}

\section{Sevugaperumal Shanmugam, Sethuri Balamurugan, Bose Raja1, Raju Nagaraj*, Kuppu Rajendran$^{1}$ and Natchimuthu Karmegam²}

${ }^{1}$ Post Graduate and Research Department of Botany, Thiagarajar College, Madurai - 625 oo9, Tamil Nadu, India

${ }^{2}$ Department of Botany, Government Arts College, Salem - 636 oo7, Tamil Nadu, India

*Corresponding author.

\begin{abstract}
A bstract
The present study includes the enumeration of floral diversity of Vaigai River in Thiruppuvanam region of Sivagangai District, was undertaken for a period of 12 months from May 2014 to April 2015. Totally 106 species belonging to 94 genera distributed among 40 families were recorded. Dicotyledons were represented by 92 species of 80 genera belonging to 35 families. Monocotyledons were 13 species of 13 genera belonging to 4 families. One pteridophyte species was also recorded in the present study. Among dicotyledons, the family Malvaceae $(8$ species) was the most dominant family. In the case of monocotyledons, Poaceae (7 species) was the dominant one. Among the 40 families listed 17 families were represented by a single genus and a single species. The herbs ( 59 species) were found to be more in number than shrub ( 27 species) followed by climber (14 species) and tree (6 species). The present study also revealed that the maximum frequency of plant population $(53.448 \%)$ was recorded for Cleome viscosa. The minimum frequency $(1.724 \%)$ was recorded for 45 plants. Maximum density of plant population was recorded for Cleome viscosa (17.986) and minimum for 21 plants $(0.035)$. The maximum abundance range (21.66) was recorded for Marsilea quadrifolia and the minimum abundance (1.00) was recorded for 25 plants.
\end{abstract}

\section{Article Info}

Accepted: 31 January 2016

Available Online: 06 February 2016

\section{Ke ywords}

Abundance

Floral diversity

Thiruppuvanam

Vaigai River

\section{Introduction}

The comprehensive studies of the plants growing in a particular area are known as flora. A flora may cover any suitable area from a small patch of forest to a Taluk, City, District, State, Country or even a Continent. Floristic studies may be a simple complied check list or an elaborate analysis of the taxa of that area. As with other parts of India, plants have been studied in Tamil $\mathrm{Nadu}$ from ancient times mainly from medicinal, agricultural, ritualistic and other utilitarian points of view. Several botanists have studied the flora of Tamil Nadu and have been able to discover many taxa new to science and also several new records. A Handbook on some South Indian weeds was prepared by Tadulingam and Venkatanarayana (1955). Flora of Presidency of Madras was published by Gamble and Fischer (1957) in 3 volumes. Some considerations on the succession of vegetation around Kodaikanal were published by MeherHomji (1969). Flora of Tamil Nadu Carnatic was also carried out (Matthew, 1981 - 83). Nair and Henry (1983) published the Flora of Tamil Nadu (Vol. I). Henry et al. (1987 and 1989) published the second and third volume of Flora of Tamilnadu respectively.

Subramanyam and Henry (1959) made a preliminary survey of the flora of Alagar hills, Karandamalais and surrounding regions in Madurai District. Krishnan and 
Sri Ganesan (1971) prepared a supplementary list of plants of Alagar hills. Selvaraj (1982) studied the floristic study of Thiruparankundram and its surroundings. Flora of Alagar hill was carried out in detail by Sri Ganesan (1984). Angiospermic flora of Pachalur hills of Dindigul district was documented by Shanmugam et al. (2010). Angiospermic herbaceous plants associated with tea plantations at Maharajanmettu in High Wavy's Mountain of Tamil Nadu were documented by Shanmugam et al. (2015). Balamurugan et al. (2015) reported the plants inhabiting in Vaigai River at Manamadurai region of Sivagangai district, Tamil Nadu. Muthupandi et al. (2015) enumerated the plants present in Vaigai River at Sakkimangalam region of Madurai district, Tamil Nadu.

The investigation about the floristic composition will definitely useful in the utilization of information by nontaxonomists like, ecological consultant, environmental engineers, forest managers, silviculturists, farmers, real estate appraisers, agricultural consultants, landscape architects, plant breeders, plant pathologists, toxicologists, forensic scientists, elementary teachers and others (Morin et al., 1988). Having these facts in mind, here an attempt is made to study the floristic diversity of Vaigai River in Thiruppuvanam region of Sivagangai district in Tamil Nadu.

\section{Materials and methods}

\section{Study area}

The floral study was conducted in Vaigai River at Thiruppuvanam and its surrounding areas which include T. Pudur, Chellappanendhal and Ladanendhal of Sivagangai district in Tamil Nadu, India. Geographically, the entire area of Thiruppuvanam is lies between $9^{\circ} 30^{\prime} \mathrm{N}$ and $9^{\circ} 42^{\prime} \mathrm{N}$ latitude and $77^{\circ} 00^{\prime} \mathrm{E}$ and $77^{\circ} 17^{\prime} \mathrm{E}$ longitude. The altitude of the study area is about $102 \mathrm{~m}$ above mean sea level. Temperature is scarcely fluctuates in the year, with the mean monthly minimum and maximum temperatures of $22^{\circ} \mathrm{C}$ and $40^{\circ} \mathrm{C}$ respectively, and annual rainfall reaches $535-800 \mathrm{~mm}$.

\section{Methodology}

A Comprehensive and exhaustive study on plants present in Vaigai River at Thiruppuvanam region was undertaken for a period of 12 months from May, 2014 to April, 2015. The known and familiar plants were recorded on the spot in the collection site itself. The unknown and doubtful plants were collected and brought to the laboratory for identification. All the plants were botanically identified by using the regional floras includes Flora of Presidency of Madras, I to III Vols. (Gamble and Fischer, 1957), Flora of Tamil Nadu Carnatic, (Matthew, 1981-83) and An Excursion Flora of Central Tamil Nadu, India (Matthew, 1991). Regarding the habit of the plants recorded during this study, prostate herb, erect herb and herb were included in the category of 'Herb'. Small shrub, sub-shrub and shrub were included in the category of 'Shrub'. Creeper, twiner and climber were mentioned as 'Climber' and small tree and trees as 'Tree'.

Quadrates of 1 sq. $m$ area were laid down in a random manner for the diversity study. Totally, 58 quadrates were included. Various species and the total number of the individuals of each species were noted and the data were considered for statistical analysis. Frequency, density and abundance (per sq. m) of the species noted were calculated using the formula by Sharma (1998).

\section{Results and discussion}

Totally 106 species (105 species of 93 genera belonging to 39 families of Angiosperms and one species of the genus belonging to a family of Pteridophyte) belonging to 94 genera distributed among 40 families were recorded. The entire list of the plants recorded in the study area is given in Table 1. Some important plants are featured in Fig. 1. Among these, dicotyledons were represented by 93 species of 81 genera belonging to 35 families and monocotyledons were 13 species of 13 genera belonging to 4 families (Table 2).

Among dicotyledons, Malvaceae was found as dominant and largest family comprising 8 species. Two families (Amaranthaceae and Aizoaceae) shared the second largest family status equally; both were represented by 7 species and considered to be co-dominant families. 4 families were represented by 6 species, 1 family was represented by 4 species, 3 families were comprising 3 species, 9 families were represented by 2 species and the rest of the families by 1 species (Table 2). In the case of monocotyledons, Poaceae was found to be dominant family comprising 7 species followed by Cyperaceae (3 species) and Commelinaceae (2 species). Pontederiaceae was represented by single species only (Table 2). Balamurugan et al. (2015) reported that regarding dicots, Asteraceae, Amaranthaceae, Euphorbiaceae and Fabaceae comprising 5 species of each and in case of monocots, Poaceae with 10 species were found as 
dominant families in Vaigai River at Manamadurai region which is adjacent to the present study area. They have documented 83 species belonging to 76 genera distributed among 37 families.

An another study carried out in Vaigai river at Sakkimangalam region of Madurai district by Muthupandi et al. (2015) revealed that totally 171 species belonging to 142 genera distributed among 55 families were recorded. Meanwhile, only 106 species were recorded by the present study. The higher number of species in Sakkimangalam region may be due the presence of Viraganoor Dam near to this area, by which the water is stored and moisture content is maintained for long time, which favors the growth of many species than that of Thiruppuvanam region.

Totally 54 herbs, 13 shrubs, 9 climbers and 7 trees were recorded from Vaigai river at Manamadurai region (Balamurugan et al., 2015) and 77 herbs (76 of angiosperm, 1 of pteridophyte), 37 shrubs, 35 trees and 23 climbers were recorded from Sakkimangalam region (Muthupandi et al., 2015). The present study revealed that herbs (59 species) were found to be more in number than shrubs (27 species) followed by climbers (14 species) and trees (6 species) (Table 1$)$.

Table 1. List of the plants recorded in the study area with their family and habit.

\begin{tabular}{|c|c|c|c|}
\hline S. No. & Botanical name & Family & Habit \\
\hline 1 & Abelmoschus setinervis Dunn & Malvaceae & Shrub \\
\hline 2 & Abrus precatorius L. ssp. precatorius & Fabaceae & Climber \\
\hline 3 & Abutilon indicum (L.) Sweet ssp. indicum & Malvaceae & Shrub \\
\hline 4 & Acalypha indica $\mathrm{L}$. & Euphorbiaceae & Herb \\
\hline 5 & Achyranthes aspera $\mathrm{L}$. & Amaranthaceae & Shrub \\
\hline 6 & Aerva lanata (L.) Juss. & Amaranthaceae & Herb \\
\hline 7 & Alternanthera sessilis (L.) R.Br. ex Dc. & Amaranthaceae & Herb \\
\hline 8 & Alysicarpus rugosus (Willd.) DC. & Fabaceae & Herb \\
\hline 9 & Amaranthus spinosus $\mathrm{L}$. & Amaranthaceae & Herb \\
\hline 10 & Ammania baccifera $\mathrm{L}$. & Lythraceae & Herb \\
\hline 11 & Annona squamosa $\mathrm{L}$. & Annonaceae & Tree \\
\hline 12 & Argemone mexicana $\mathrm{L}$. & Papaveraceae & Herb \\
\hline 13 & Asystasia gangetica (L.) T.Anderson & Acanthaceae & Shrub \\
\hline 14 & Azadirachta indica Adr. Juss & Meliaceae & Tree \\
\hline 15 & Baccopa monnieri (L.) Pennell & Scrophulariaceae & Herb \\
\hline 16 & Bambusa arundinacea (Retz.) Willd. & Poaceae & Herb \\
\hline 17 & Boerhavia diffusa $\mathrm{L}$. & Nyctaginaceae & Herb \\
\hline 18 & Boerhavia erecta $\mathrm{L}$. & Nyctaginaceae & Herb \\
\hline 19 & Calotropis gigantea (L.) R.Br. & Asclepiadaceae & Shrub \\
\hline 20 & Cardiospermum halicacabum $\mathrm{L}$. & Sapindaceae & Climber \\
\hline 21 & Canavalia virosa (Roxb.) Wight \& Arn. & Fabaceae & Climber \\
\hline 22 & Celosia cristata L. Kuntze & Amaranthaceae & Shrub \\
\hline 23 & Chromolaena odorata $\mathrm{L}$. & Asteraceae & Shrub \\
\hline 24 & Cissus setosa Wallich & Vitaceae & Climber \\
\hline 25 & Citrullus colocynthis (L.) Schader & Cucurbitaceae & Climber \\
\hline 26 & Chloris barbata $\mathrm{Sw}$. & Poaceae & Herb \\
\hline 27 & Cleome viscosa $\mathrm{L}$. & Capparidaceae & Herb \\
\hline 28 & Coccinia grandis (L.) J.Voigt & Cucurbitaceae & Climber \\
\hline 29 & Commelina benghalensis L. & Commelinaceae & Herb \\
\hline 30 & Corchorus trilocularis $\mathrm{L}$. & Tiliaceae & Shrub \\
\hline 31 & Croton bonplandianus Baillon & Euphorbiaceae & Shrub \\
\hline 32 & Cucumis melo $\mathrm{L}$. & Cucurbitaceae & Climber \\
\hline 33 & Cynodon dactylon (L.) Pers. & Poaceae & Herb \\
\hline 34 & Cyanotis arachnoidea C.B. Clarke & Commelinaceae & Herb \\
\hline 35 & Cyperus rotundus $\mathrm{L}$. & Cyperaceae & Herb \\
\hline 36 & Datura metel L. & Solanaceae & Shrub \\
\hline 37 & Diptercanthus patulus (Jacq.) Nees & Acanthaceae & Herb \\
\hline
\end{tabular}




\begin{tabular}{|c|c|c|c|}
\hline S. No. & Botanical name & Family & Habit \\
\hline 38 & Eclipta prostrata (L.) L. & Asteraceae & Herb \\
\hline 39 & Eichhornia crassipes (C. Martius) Salms-Laub & Pontederiaceae & Herb \\
\hline 40 & Eragrostis unioloides Steudel & Poaceae & Herb \\
\hline 41 & Euphorbia hirta L. & Euphorbiaceae & Herb \\
\hline 42 & Ficus religiosa $\mathrm{L}$. & Moraceae & Tree \\
\hline 43 & Fimbristylis eragrostis (Nees) Hance & Cyperaceae & Herb \\
\hline 44 & Glinus lotoides L. & Aizoaceae & Herb \\
\hline 45 & Glinus oppositifolius (L.) DC. & Aizoaceae & Herb \\
\hline 46 & Gomphrena celosiodes $\mathrm{C}$. Martius & Amaranthaceae & Herb \\
\hline 47 & Heliotropium indicum $\mathrm{L}$. & Boraginaceae & Herb \\
\hline 48 & Hibiscus micranthus L.f. & Malvaceae & Shrub \\
\hline 49 & Hibiscus vitifolius $\mathrm{L}$. & Malvaceae & Shrub \\
\hline 50 & Hybanthus enneaspermus (L.) F.Muell & Violaceae & Herb \\
\hline 51 & Indigofera linnaei Ali. & Fabaceae & Herb \\
\hline 52 & Ipomoea carnea Jacq. ssp. fistulosa (Choisy) D. Austin & Convolvulaceae & Shrub \\
\hline 53 & Ipomoea pes-tigridis $\mathrm{L}$. & Convolvulaceae & Climber \\
\hline 54 & Justicia tranquebariensis L.f. & Acanthaceae & Shrub \\
\hline 55 & Lantana camara $\mathrm{L}$. & Verbenaceae & Shrub \\
\hline 56 & Leonotis nepetiifolia (L.) R.Br. & Lamiaceae & Herb \\
\hline 57 & Leucaena leucocephala (Lam.) de Wit & Mimosaceae & Tree \\
\hline 58 & Leucas aspera (Willd.) Link & Lamiaceae & Herb \\
\hline 59 & Luffa cylindrica (L.) M. Roemer & Cucurbitaceae & Climber \\
\hline 60 & Marsilea quardrifolia $\mathrm{L}$. & Marsiliaceae & Herb \\
\hline 61 & Mollugo cerviana (L.) Ser. var. spathulifolia Fenzc & Aizoaceae & Herb \\
\hline 62 & Mollugo nudicaulis Lam. & Aizoaceae & Herb \\
\hline 63 & Mollugo pentaphylla $\mathrm{L}$. & Aizoaceae & Herb \\
\hline 64 & Ocimum canum Sims & Lamiaceae & Herb \\
\hline 65 & Oldenlandia umbellata $\mathrm{L}$. & Rubiaceae & Herb \\
\hline 66 & Opuntia dillenii (Ker Gawler) Haw. & Cactaceae & Shrub \\
\hline 67 & Oxystelma esculentum (L.f.) R.Br. ex. Schultes & Asclepiadaceae & Climber \\
\hline 68 & Panicum repens $\mathrm{L}$. & Poaceae & Herb \\
\hline 69 & Parthenium hysterophorus L. & Asteraceae & Herb \\
\hline 70 & Passiflora edulis Sims & Passifloraceae & Climber \\
\hline 71 & Passiflora foetida $\mathrm{L}$. & Passifloraceae & Climber \\
\hline 72 & Pedalium murex $\mathrm{L}$ & Pedaliaceae & Herb \\
\hline 73 & Peristophe paniculata (Forsskal) Brummit. & Acanthaceae & Herb \\
\hline 74 & Pergularia daemia (Forsskal) Chiov. & Asclepiadaceae & Climber \\
\hline 75 & Phaseolus trilobus auct.non L. & Fabaceae & Herb \\
\hline 76 & Phyla nodiflora (L.) E. Greene & Verbenaceae & Herb \\
\hline 77 & Phyllanthus amarus Schum \& Thonn. & Euphorbiaceae & Herb \\
\hline 78 & Phyllanthus maderaspatensis $\mathrm{L}$. & Euphorbiaceae & Herb \\
\hline 79 & Physalis minima $\mathrm{L}$. & Solanaceae & Shrub \\
\hline 80 & Pithecellobium dulce (Roxb.) Benth. & Mimosaceae & Tree \\
\hline 81 & Portulaca oleracea Willd. & Portulacaceae & Shrub \\
\hline 82 & Prosopis juliflora (Sw.) DC. & Mimosaceae & Tree \\
\hline 83 & Pupalia lappacea (L.) A.L. Juss. var. lappacea & Amaranthaceae & Herb \\
\hline 84 & Ricinus communis (L.) Nees & Euphorbiaceae & Shrub \\
\hline 85 & Rungia repens $(\mathrm{L}$.$) Nees$ & Acanthaceae & Herb \\
\hline 86 & Ruellia tuberosa $\mathrm{L}$. & Acanthaceae & Herb \\
\hline 87 & Saccharum spontaneum $\mathrm{L}$. & Poaceae & Shrub \\
\hline 88 & Scirpus grossus L.f. & Cyperaceae & Herb \\
\hline 89 & Scoparia dulcis L. & Scrophulariaceae & Herb \\
\hline
\end{tabular}




\begin{tabular}{clll}
\hline S. No. & Botanical name & Family & Habit \\
\hline 90 & Setaria italica (L.) P. Beauv. & Poaceae & Herb \\
91 & Sida acuta Burm. f. & Malvaceae & Shrub \\
92 & Sida cordata L. & Malvaceae & Shrub \\
93 & Sida cordifolia L. & Malvaceae & Shrub \\
94 & Sida rhombifolia L. & Malvaceae & Shrub \\
95 & Spermacoce hispida L. & Rubiaceae & Herb \\
96 & Streblus asper Lour. & Moraceae & Shrub \\
97 & Tephrosia purpurea (L.) Pers. & Fabaceae & Shrub \\
98 & Tinospora cordifolia Hook.f. \& Thomson & Menispermaceae & Climber \\
99 & Trianthema decandra L. & Aizoaceae & Herb \\
100 & Trianthema portulacastrum L. & Aizoaceae & Herb \\
101 & Tribulus terrestris L. & Zygophyllaceae & Herb \\
102 & Trichodesma indicum (L.) R.Br. & Boraginaceae & Herb \\
103 & Tridax procumbens L. & Asteraceae & Herb \\
104 & Vernonia cinerea (L.) Less. & Asteraceae & Herb \\
105 & Waltheria indica L. & Sterculiaceae & Shrub \\
106 & Xanthium indicum J. Koenig & Asteraceae & Herb \\
\hline
\end{tabular}
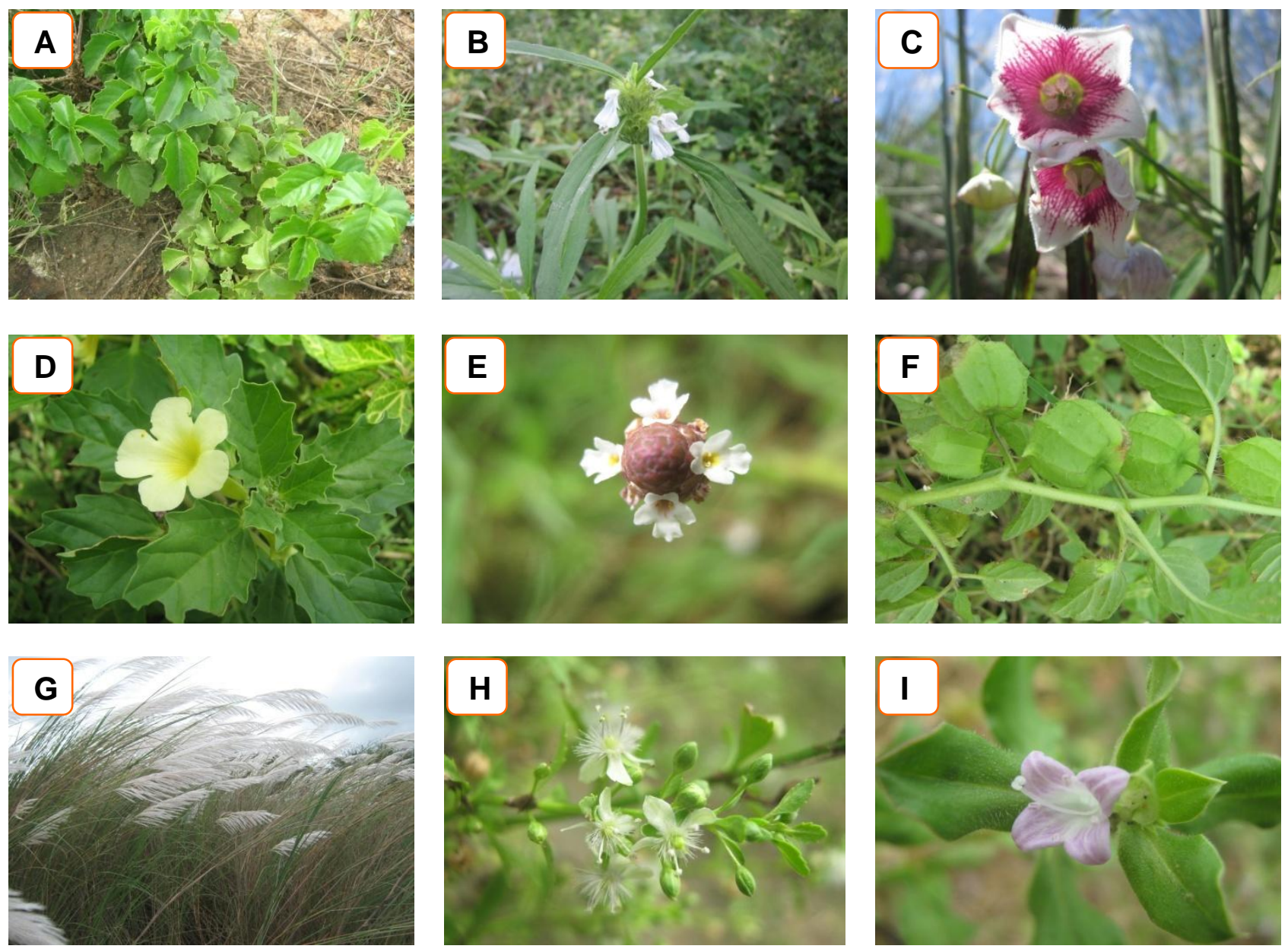

Fig. 1: Some important plants recorded during the survey. (A) Cissus setosa, (B) Leucas aspera, (C) Oxystelma esculentum, (D) Pedalium murex, (E) Phyla nodiflora, (F) Physalis minima, (G) Saccharum spontaneum, (H) Scoparia dulcis and (I) Spermacoce hispida. 
Table 2. List of families of the plants with number of genera and species.

\begin{tabular}{|c|c|c|c|}
\hline S. No. & Family name & No. of genera & No. of species \\
\hline 1 & Acanthaceae & 6 & 6 \\
\hline 2 & Aizoaceae & 3 & 7 \\
\hline 3 & Amaranthaceae & 7 & 7 \\
\hline 4 & Asteraceae & 1 & 1 \\
\hline 5 & Asclepiadaceae & 3 & 3 \\
\hline 6 & Asteraceae & 6 & 6 \\
\hline 7 & Boraginaceae & 2 & 2 \\
\hline 8 & Cactaceae & 1 & 1 \\
\hline 9 & Capparidaceae & 1 & 1 \\
\hline 10 & Commelinaceae* & 2 & 2 \\
\hline 11 & Convolvulaceae & 1 & 2 \\
\hline 12 & Cucurbitaceae & 4 & 4 \\
\hline 13 & Cyperaceae* & 3 & 3 \\
\hline 14 & Euphorbiaceae & 5 & 6 \\
\hline 15 & Fabaceae & 6 & 6 \\
\hline 16 & Lamiaceae & 3 & 3 \\
\hline 17 & Lythraceae & 1 & 1 \\
\hline 18 & Malvaceae & 4 & 8 \\
\hline 19 & Marsiliaceae $^{\dagger}$ & 1 & 1 \\
\hline 20 & Meliaceae & 1 & 1 \\
\hline 21 & Menispermaceae & 1 & 1 \\
\hline 22 & Mimosaceae & 3 & 3 \\
\hline 23 & Moraceae & 2 & 2 \\
\hline 24 & Nyctaginaceae & 1 & 2 \\
\hline 25 & Papaveraceae & 1 & 1 \\
\hline 26 & Passifloraceae & 1 & 2 \\
\hline 27 & Pedaliaceae & 1 & 1 \\
\hline 28 & Poaceae* & 7 & 7 \\
\hline 29 & Pontederiaceae* & 1 & 1 \\
\hline 30 & Portulacaceae & 1 & 1 \\
\hline 31 & Rubiaceae & 2 & 2 \\
\hline 32 & Sapindaceae & 1 & 1 \\
\hline 33 & Scrophulariaceae & 2 & 2 \\
\hline 34 & Solanaceae & 2 & 2 \\
\hline 35 & Sterculiaceae & 1 & 1 \\
\hline 36 & Tiliaceae & 1 & 1 \\
\hline 37 & Verbenaceae & 2 & 2 \\
\hline 38 & Violaceae & 1 & 1 \\
\hline 39 & Vitaceae & 1 & 1 \\
\hline 40 & Zygophyllaceae & 1 & 1 \\
\hline
\end{tabular}

*Monocot; ${ }^{\dagger}$ Pteridophyte; Others - Dicotyledons.

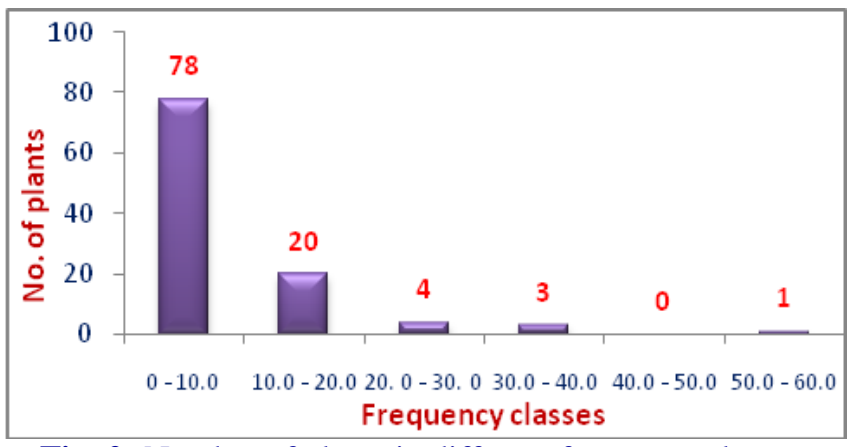

Fig. 2: Number of plants in different frequency classes.

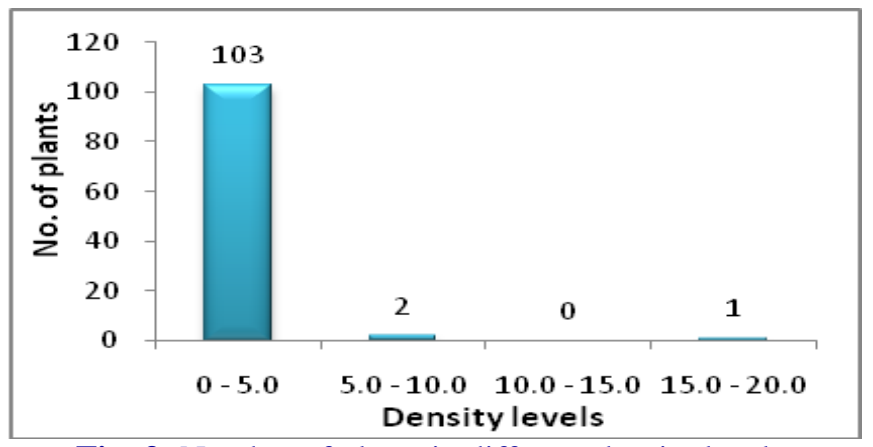

Fig. 3: Number of plants in different density levels. 
In the case of dicotyledons, among 93 species recorded 59 species were found to be herbs, 14 species were found to be shrubs, 14 species were found to be climbers and 6 species were trees. Among monocots, 11 were herbs, 1 was shrub and 1 was tree. Regarding the trees found in the Vaigai River, Annona squamosa, Azadirachta indica, Bambusa arundinacea, Ficus religiosa, Leucaena leucocephala, Pithecellobium dulce and Prosopis juliflora are being in seedling stage as their mature trees are present in the river bank. Prosopis juliflora is being in mature stage also.

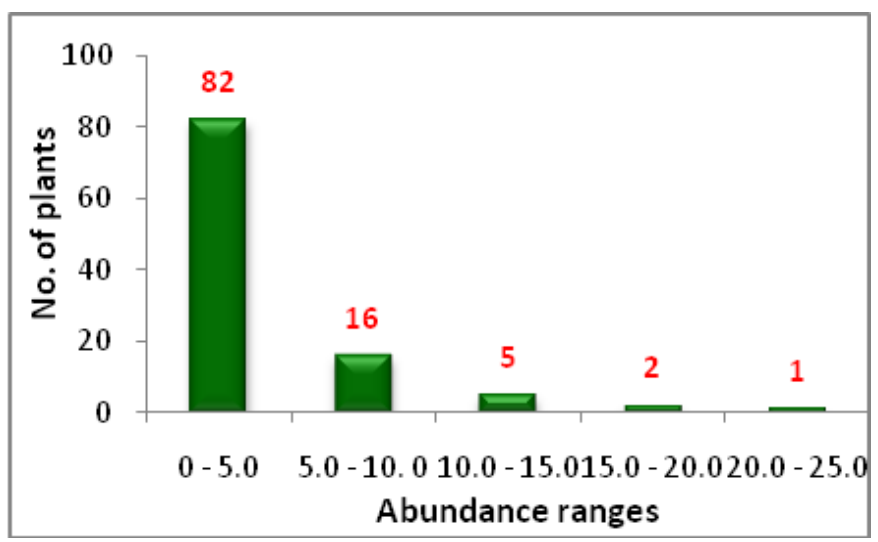

Fig. 4: Number of plants in different abundance ranges.
From the present investigation it was found that, among 106 plants recorded, about 78, 20, 4, 3 and 1 number of species were recorded at $0-10.0,10.0-20.0,20.0-30.0$, $30.0-40.0$ and $50.0-60.0 \%$ of frequency range respectively (Fig. 2). The maximum frequency of plant population $(53.448 \%)$ was recorded for Cleome viscosa followed by Cynodon dactylon (36.206\%). The minimum frequency (1.724\%) was recorded for 45 plants (Table 3 ). Balamurugan (2012) stated that maximum frequency (69.23\%) was recorded for Cynodon dactylon followed by Oldenlandia umbellata (53.84\%), Prosopis juliflora (50\%) and Eragrostis unioloides (42.30\%) and minimum frequency $(3.84 \%)$ was recorded for 39 plants. It was also found that the density of the plant species varied considerably. Maximum density of plant population was recorded for Cleome viscosa (17.986) and minimum for 21 plants (0.035). 103, 2, and 1 number of plants were recorded at $0-5.0,5.0-10.0$ and $15.0-20.0$ density level respectively (Fig. 3). Regarding abundance, about $82,16,5,2$ and 1 number of species were recorded at $0-$ 5.0, $5.0-10.0,10.0-15.0,15.0-20.0$ and $20.0-25.0$ of abundance range respectively (Fig. 4). The maximum abundance range (21.66) was recorded for Marsilea quadrifolia and the minimum abundance (1.00) was recorded for 25 plants (Table 3 ).

Table 3. Plants with their frequency, density and abundance.

\begin{tabular}{cllll}
\hline S. No & Botanical name & Frequency & Density & Abundance \\
\hline 1 & Abelmoschus setinervis Dunn & 0.724 & 0.107 & 3.0 \\
2 & Abrus precatorius L. ssp. Precatorius & 0.724 & 0.071 & 2.0 \\
3 & Abutilon indicum (L.) Sweet ssp. Indicum & 5.172 & 0.322 & 3.0 \\
4 & Acalypha indica L. & 22.413 & 2.794 & 6.0 \\
5 & Achyranthes aspera L. & 3.448 & 0.143 & 2.0 \\
6 & Aerva lanata (L.) Juss. & 1.724 & 0.107 & 3.0 \\
7 & Alternanthera sessilis (L.) R.Br. ex Dc. & 22.413 & 1.469 & 3.15 \\
8 & Alysicarpus rugosus (Willd.) DC. & 1.724 & 0.107 & 3.0 \\
9 & Amaranthus spinosus L. & 5.172 & 0.250 & 2.333 \\
10 & Ammania baccifera L. & 6.896 & 0.394 & 2.75 \\
11 & Annona squamosa L. & 1.724 & 0.071 & 2.0 \\
12 & Argemone mexicana L. & 1.724 & 0.107 & 3.0 \\
13 & Asystasia gangetica (L.) T.Anderson & 1.724 & 0.107 & 3.0 \\
14 & Azadirachta indica Adr. Juss & 6.896 & 0.895 & 6.25 \\
15 & Baccopa monnieri (L.) Pennell & 3.448 & 0.537 & 7.5 \\
16 & Bambusa arundinacea (Retz.) Willd. & 5.172 & 0.358 & 3.333 \\
17 & Boerhavia diffusa L. & 13.793 & 0.859 & 3.0 \\
18 & Boerhavia erecta L. & 15.519 & 4.836 & 15 \\
19 & Calotropis gigantea (L.) R.Br. & 13.793 & 0.286 & 1.0 \\
20 & Cardiospermum halicacabum L. & 3.448 & 0.143 & 2.0 \\
21 & Canavalia virosa (Roxb.) Wight $\&$ Arn. & 1.724 & 0.035 & 1.0 \\
22 & Celosia cristata L. Kuntze & 1.724 & 0.143 & 4.0 \\
23 & Chromolaena odorata L. & 1.724 & 0.035 & 1.0 \\
24 & Cissus setosa Wallich & 6.896 & 0.394 & 2.75 \\
25 & Citrullus colocynthis (L.) Schader & 12.068 & 1.218 & 4.85 \\
26 & Chloris barbata Sw. & 53.448 & 17.986 & 16.19 \\
\hline
\end{tabular}




\begin{tabular}{|c|c|c|c|c|}
\hline S. No & Botanical name & Frequency & Density & Abundance \\
\hline 27 & Cleome viscosa $\mathrm{L}$. & 10.344 & 0.286 & 1.33 \\
\hline 28 & Coccinia grandis (L.) J.Voigt & 3.448 & 0.143 & 2.0 \\
\hline 29 & Commelina benghalensis L. & 3.448 & 0.107 & 1.5 \\
\hline 30 & Corchorus trilocularis L. & 0.448 & 1.218 & 5.66 \\
\hline 31 & Croton bonplandianus Baillon & 1.724 & 0.035 & 1.0 \\
\hline 32 & Cucumis melo $\mathrm{L}$. & 36.206 & 9.278 & 12.33 \\
\hline 33 & Cynodon dactylon (L.) Pers. & 1.724 & 0.035 & 1.0 \\
\hline 34 & Cyanotis arachnoidea C.B. Clarke & 10.344 & 2.973 & 13.83 \\
\hline 35 & Cyperus rotundus $\mathrm{L}$. & 3.448 & 0.071 & 1.0 \\
\hline 36 & Datura metel L. & 1.724 & 0.179 & 5.0 \\
\hline 37 & Diptercanthus patulus (Jacq.) Nees & 17.241 & 3.332 & 9.3 \\
\hline 38 & Eclipta prostrata $($ L.) L. & 1.724 & 0.609 & 17 \\
\hline 39 & Eichhornia crassipes (C. Martius) Salms-Laub & 0.244 & 2.651 & 12.33 \\
\hline 40 & Eragrostis unioloides Steudel & 13.793 & 1.361 & 4.75 \\
\hline 41 & Euphorbia hirta L. & 1.724 & 0.035 & 1.0 \\
\hline 42 & Ficus religiosa $\mathrm{L}$. & 6.896 & 1.003 & 7.0 \\
\hline 43 & Fimbristylis eragrostis (Nees) Hance & 1.724 & 0.035 & 1.0 \\
\hline 44 & Glinus lotoides L. & 1.724 & 0.071 & 2.0 \\
\hline 45 & Glinus oppositifolius (L.) DC. & 3.448 & 0.286 & 4.0 \\
\hline 46 & Gomphrena celosiodes C. Martius & 1.724 & 0.035 & 1.0 \\
\hline 47 & Heliotropium indicum $\mathrm{L}$. & 1.724 & 0.035 & 1.0 \\
\hline 48 & Hibiscus micranthus L.f. & 5.172 & 0.322 & 3.0 \\
\hline 49 & Hibiscus vitifolius $\mathrm{L}$. & 1.724 & 0.035 & 1.0 \\
\hline 50 & Hybanthus enneaspermus (L.) F.Muell & 1.724 & 0.071 & 2.0 \\
\hline 51 & Indigofera linnaei Ali. & 6.896 & 0.358 & 2.5 \\
\hline 52 & Ipomoea carnea Jacq. ssp. fistulosa (Choisy) D. Austin & 1.724 & 0.035 & 1.0 \\
\hline 53 & Ipomoea pes-tigridis L. & 0.344 & 0.429 & 2.0 \\
\hline 54 & Justicia tranquebariensis L.f. & 1.724 & 0.035 & 1.0 \\
\hline 55 & Lantana camara $\mathrm{L}$ & 1.724 & 0.250 & 7.0 \\
\hline 56 & Leonotis nepetiifolia (L.) R.Br. & 1.724 & 0.250 & 7.0 \\
\hline 57 & Leucaena leucocephala (Lam.) de Wit & 1.724 & 0.035 & 1.0 \\
\hline 58 & Leucas aspera (Willd.) Link & 6.896 & 0.143 & 1.0 \\
\hline 59 & Luffa cylindrica (L.) M. Roemer & 5.172 & 2.328 & 21.66 \\
\hline 60 & Marsilea quardrifolia $\mathrm{L}$. & 6.896 & 0.931 & 6.5 \\
\hline 61 & Mollugo cerviana (L.) Ser. var. spathulifolia Fenzc & 6.896 & 2.221 & 4.76 \\
\hline 62 & Mollugo nudicaulis Lam. & 22.413 & 2.543 & 4.73 \\
\hline 63 & Mollugo pentaphylla L. & 1.724 & 0.071 & 2.0 \\
\hline 64 & Ocimum canum Sims & 8.620 & 0.609 & 3.4 \\
\hline 65 & Oldenlandia umbellata $\mathrm{L}$. & 1.724 & 0.107 & 3.0 \\
\hline 66 & Opuntia dillenii (Ker Gawler) Haw. & 1.724 & 0.035 & 1.0 \\
\hline 67 & Oxystelma esculentum (L.f.) R.Br. ex. Schultes & 10.344 & 1.719 & 8.0 \\
\hline 68 & Panicum repens $\mathrm{L}$. & 31.034 & 4.192 & 6.5 \\
\hline 69 & Parthenium hysterophorus L. & 1.724 & 0.035 & 1.0 \\
\hline 70 & Passiflora edulis Sims & 8.620 & 0.214 & 1.2 \\
\hline 71 & Passiflora foetida $\mathrm{L}$. & 1.724 & 0.179 & 5.0 \\
\hline 72 & Pedalium murex $\mathrm{L}$. & 5.172 & 0.358 & 3.33 \\
\hline 73 & Peristophe paniculata (Forsskal) Brummit. & 10.344 & 0.214 & 1.0 \\
\hline 74 & Pergularia daemia (Forsskal) Chiov. & 1.724 & 0.035 & 1.0 \\
\hline 75 & Phaseolus trilobus auct.non L. & 15.517 & 2.50 & 7.77 \\
\hline 76 & Phyla nodiflora (L.) E.Greene & 10.344 & 1.182 & 5.5 \\
\hline 77 & Phyllanthus amarus Schum \& Thonn. & 8.620 & 0.573 & 3.2 \\
\hline 78 & Phyllanthus maderaspatensis $\mathrm{L}$. & 5.172 & 0.179 & 1.66 \\
\hline 79 & Physalis minima $\mathrm{L}$. & 3.448 & 0.107 & 1.5 \\
\hline 80 & Pithecellobium dulce (Roxb.) Benth. & 10.344 & 0.102 & 19.5 \\
\hline 81 & Portulaca oleracea Willd. & 17.241 & 0.967 & 2.7 \\
\hline 82 & Prosopis juliflora (Sw.) DC. & 1.724 & 0.035 & 1.0 \\
\hline
\end{tabular}




\begin{tabular}{cllll}
\hline S. No & Botanical name & Frequency & Density & Abundance \\
\hline 83 & Pupalia lappacea (L.) A.L. Juss. var. lappacea & 3.448 & 0.107 & 1.5 \\
84 & Ricinus communis (L.) Nees & 1.724 & 0.035 & 1.0 \\
85 & Rungia repens (L.) Nees & 1.724 & 0.035 & 1.0 \\
86 & Ruellia tuberos L. & 32.758 & 7.703 & 11.31 \\
87 & Saccharum spontaneum L. & 1.724 & 0.322 & 9.0 \\
88 & Scirpus grossus L.f. & 3.448 & 0.214 & 3.0 \\
89 & Scoparia dulcis L. & 1.724 & 0.250 & 7.0 \\
90 & Setaria italica (L.) P. Beauv. & 3.448 & 0.143 & 2.0 \\
91 & Sida acuta Burm. f. & 1.724 & 0.035 & 1.0 \\
92 & Sida cordata L. & 1.724 & 0.035 & 1.0 \\
93 & Sida cordifolia L. & 6.896 & 0.358 & 2.5 \\
94 & Sida rhombifolia L. & 5.172 & 0.179 & 1.66 \\
95 & Spermacoce hispida L. & 1.724 & 0.071 & 2.0 \\
96 & Streblus asper Lour. & 1.724 & 0.107 & 3.0 \\
97 & Tephrosia purpurea (L.) Pers. & 5.172 & 0.250 & 2.33 \\
98 & Tinospora cordifolia Hook.f. \& Thomson & 1.724 & 0.609 & 17 \\
99 & Trianthema decandra L. & 8.620 & 0.501 & 2.8 \\
100 & Trianthema portulacastrum L. & 13.793 & 1.683 & 5.87 \\
101 & Tribulus terrestris L. & 3.448 & 0.107 & 1.5 \\
102 & Trichodesma indicum (L.) R.Br. & 10.344 & 1.003 & 4.66 \\
103 & Tridax procumbens L. & 1.724 & 0.071 & 2.0 \\
104 & Vernonia cinerea L.) Less. & 1.724 & 0.035 & 1.0 \\
105 & Waltheria indica L. & 18.965 & 0.931 & 2.36 \\
106 & Xanthium indicum J. Koenig & 1.724 & 0.107 & 3.0 \\
\hline
\end{tabular}

\section{Conclusion}

In the present study, totally 106 species belonging to 94 genera distributed among 40 families were recorded. Among dicotyledons, Malvaceae was the dominant family comprising 8 species whereas, Poaceae was found to be dominant family in monocotyledons. The herbs were found to be more in number than shrubs. The plant population frequency was higher for Cleome viscosa (53.448\%) followed by Cynodon dactylon $(36.206 \%)$. The density of the plant species enumerated in the study varied considerably. Further studies are required to be carried out to explore the entire floral wealth of Vaigai River and conservation strategies must be created on plants available with less frequency. Otherwise there may a possibility of the extinction of any particular plant species.

\section{Conflict of interest statement}

Authors declare that they have no conflict of interest.

\section{Acknowledgement}

Two of us (SS \& RN) are cordially grateful to Mr. M. Kaveri, who has helped us in plant collection during field visits. One of us (SS) is thankful to University Grants Commission, New Delhi for financial Assistance under Rajiv Gandhi National Fellowship Scheme, 2008.

\section{References}

Balamurugan, S., 2012. Floral diversity of Vaigai River and their utilization as medicine in Manamadurai region of Sivagangai district in Tamil Nadu. M.Sc., Dissertation, Thiagarajar College, Madurai, Tamil Nadu.

Balamurugan, S., Shanmugam, S., Muthupandi, C.P., Suresh, K., Rajendran, K., 2015. Floral diversity of Vaigai river in Manamadurai region of Sivagangai district in Tamil Nadu, Southern India. In: Biodiversity \& Evaluation - Perspective and Paradigm shifts. Department of Zoology, Sree Sankara College, Kalady, Kerala. pp. 217-219.

Gamble, J.S., Fischer, C.E.C., 1957. The Flora of the Presidency of Madras. Vols. I-III, Botanical survey of India, Kolkata.

Henry, A.N., Chitra, V., Balakrishnan, N.P., 1989. Flora of Tamil Nadu, India, Series-I, Analysis Volume III, Botanical Survey of India, Southern Circle, Coimbatore.

Henry, A.N., Kumari, G.R., Chitra, V., 1987. Flora of Tamil Nadu, India, Series-I, Analysis Volume II, Botanical Survey of India, Southern Circle, Coimbatore.

Krishnan, S., Sri Ganesan, T., 1971. Flora of Alagar Hills. Suppl. List. J. Madurai Univer. 3(1), 50-53.

Matthew, K.M., 1981 - 83. Flora of Tamil Nadu Carnatic. The Rapinat Herbarium, St. Joseph's College, Tiruchirapalli, Tamil Nadu. India.

Matthew, K.M., 1991. An Excursion Flora of Central Tamil Nadu. Oxford - IBH Publishing Co. Pvt. Ltd., New Delhi. India. 
Meher-Homji, V.M., 1969. Some considerations on the succession on vegetation around Kodaikanal. J. Indian Bot. Soc. 48, 43-52.

Muthupandi, C. P., Shanmugam, S., Balamurugan, S., Rajendran, K., 2015. Floral diversity of Vaigai river in Sakkimangalam region of Madurai district in Tamil Nadu, Southern India. In: Biodiversity \& Evaluation Perspective and Paradigm shifts. Department of Zoology, Sree Sankara College, Kalady, Kerala. pp. 233-236.

Nair, N.C., Henry, A.N., 1983. Flora of Tamil Nadu, India, Series-I, Analysis volume I, Botanical survey of India, Southern Circle, Coimbatore.

Selvaraj, A., 1982. A Floristic Study of Thirupparankundram and its surroundings. M.Sc. Dissertation, Thiagarajar College, Madurai, Tamil Nadu, India.

Shanmugam, S., Selvakumar, P., Mohan, S., Dinesh Kumar, G., Rajendran, K., 2010. Angiospermic flora of Pachalur hills in Dindigul district of Tamil Nadu, India - A Checklist. J. Phytol. Res. 23(2), 223-238.
Shanmugam, S., Raja, B., Balamurugan, S., Muthupandi, C.P., Rajendran, K., 2015. Angiospermic herbaceous plants associated with tea plantations at Maharajanmettu in High Wavy's Mountain of Tamil Nadu, India. In: Biodiversity \& Evaluation - Perspective and Paradigm shifts. Department of Zoology, Sree Sankara College, Kalady, Kerala. pp. 214-216.

Sharma, P.D., 1998. Ecology and Environment, Rastogi Publication, Meerut. India.

Sri Ganesan, T., 1984. Flora of Alagar Hills. Ph.D. Thesis, Madurai Kamaraj University, Madurai, Tamil Nadu, India.

Subramaniyam, K., Henry, A.N., 1959. A Contribution to the flora of Alagar hills, Karandamalais and surrounding regions in Madurai District, Madras State. J. Indian Bot. Soc. 38, 492-527.

Tadulingam, C., Venkatanarayana, G., 1955. A Hand book of some South Indian Weeds. Govt. Press, Madras.

\section{How to cite this article:}

Shanmugam, S., Balamurugan, S., Raja, B., Nagaraj, R., Rajendran, K., Karmegam, N., 2016. Floral diversity of Vaigai River in Thiruppuvanam region of Sivagangai district, Tamil Nadu, southern India. Int. J. Curr. Res. Biosci. Plant Biol. 3(2), 96-105.

doi: http://dx.doi.org/10.20546/ijcrbp.2016.302.012 